\title{
Use of cement combined grafting in upper and lower extremity benign bone tumors
}

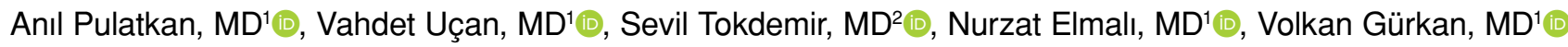 \\ ${ }^{1}$ Department of Orthopedics and Traumatology, Bezmialem Vakıf University School of Medicine, Istanbul, Turkey \\ ${ }^{2}$ Department of Radiology, Bezmialem Vakıf University School of Medicine, Istanbul, Turkey
}

There is no standard procedure for the treatment of benign bone tumors. The bone defect following the curettage of the bone tumor can be filled with autologous bone marrow, polymethylmethacrylate cement, allograft, tricalcium phosphate, and demineralized bone matrix (DBM). All these procedures have their own advantages and disadvantages. Autografting is the gold standard in tumor surgery; nevertheless, its disadvantages including limited access, cosmetic problems, and donor site morbidity make the alternative treatment modalities as viable options. Resorption of graft material and transmission of disease are associated risks of allograft use. ${ }^{[1]}$ Polymethylmethacrylate cement is non-biological and its Young's modulus of elasticity is lower than cortical bone, responds to compression-distraction forces differently compared with cortical bone, and has poor tensile and shear strength. ${ }^{[2]}$ Demineralized bone matrix is expensive and osteoinductive without structural support. ${ }^{[3]}$

Our hypothesis was that cement combined DBM treatment stimulates new bone formation, thus improves the functional scores. To the authors' knowledge, no study has focused on this technique

Received: November 08, 2019

Accepted: December 15, 2019

Published online: June 18, 2020

Correspondence: Anıl Pulatkan, MD. Bezmi Alem Vakıf Üniversitesi Tıp Fakültesi Ortopedi ve Travmatoloji Anabilim Dalı, 34093 Fatih, İstanbul, Türkiye.

E-mail: anilpulatkan@gmail.com

Doi: 10.5606/ehc. 2020.71918

Citation: Pulatkan A, Uçan V, Tokdemir S, Elmalı N, Gürkan V. Use of cement combined grafting in upper and lower extremity benign bone tumors. Jt Dis Relat Surg 2020;31(2):335-340.

\section{ABSTRACT}

Objectives: This study aims to investigate the effectivity of cement combined demineralized bone matrix (DBM) treatment on new bone formation in the cortical window as well as to evaluate the effect of new bone formation on functional outcomes.

Patients and methods: Thirty-two benign bone tumor patients (15 males, 17 females; median age 38 years; range, 12 to 68 years), who were treated with cement combined DBM between February 2010 and December 2014, were evaluated retrospectively. Patient characteristics were recorded as age, gender, tumor localization, histological diagnosis, Enneking stage, tumor size, size of the cortical window, usage of prophylactic fixation, time to return to work, Musculoskeletal Tumor Society (MSTS) functional score, tumor relapse, and new bone formation on the cortical window in the computed tomography scans after one year of surgery.

Results: Median tumor volume was $17.2 \mathrm{~cm}^{3}$ (range, 2.8 to $139.6 \mathrm{~cm}^{3}$ ), median area of the cortical window was $8.3 \mathrm{~cm}^{2}$ (range, 1.6 to $28.4 \mathrm{~cm}^{2}$ ), and median postoperative one-year MSTS score was 84.5 (range, 66 to 97). MSTS scores were significantly worse with the usage of prophylactic fixation $(\mathrm{p}<0.001)$. There was a statistically significant difference between the usage of prophylactic fixation and cortical window size $(p=0.013)$. There was a low-level negative correlation in terms of age and bone formation on the cortical window $(\mathrm{p}=0.046, \mathrm{r}=-0.356)$ and mid-level negative correlation between cortical window size and functional scores $(\mathrm{p}=0.001, \mathrm{r}=-0.577)$.

Conclusion: Application of cement combined with DBM procedure is an effective, alternative, and biological treatment in bone tumors that provides immediate stability and stimulates new bone formation on the cortical window.

Keywords: Benign bone tumor, cement, demineralized bone matrix.

and searched the effect of new bone formation in the cortical window on functional outcomes. Therefore, in this study, we aimed to investigate the effectivity of cement combined DBM treatment on new bone formation in the cortical window as well as to evaluate the effect of new bone formation on functional outcomes. 


\section{PATIENTS AND METHODS}

Thirty-two benign bone tumor patients (15 males, 17 females; median age 38 years; range, 12 to 68 years), who underwent cement combined DBM procedure at Bezmialem Vakıf University School of Medicine between February 2010 and December 2014 and were followed up for a minimum of one year, were evaluated retrospectively. Patients with axial $(n=2)$, pelvic bone tumors $(n=3)$, metastatic giant cell bone tumor $(n=2)$, or those who underwent adjuvant radiotherapy or chemotherapy $(\mathrm{n}=1)$ or were followed up for less than one year $(n=11)$ were excluded. The study protocol was approved by the Bezmialem Vakıf University School of Medicine Ethics Committee. A written informed consent was obtained from each patient. The study was conducted in accordance with the principles of the Declaration of Helsinki.

The mean follow-up time was $20.8 \pm 7.7$ months. There were simple bone cysts $(\mathrm{n}=6,19 \%)$, enchondromas $(n=14,43 \%)$, aneurysmal bone cyst $(n=1,3 \%)$, fibrous dysplasia $(n=3,9 \%)$, chondroblastomas $(n=2,6 \%)$, and giant cell bone tumors $(n=6,19 \%)$ according to the pathology results.

The lesions were located at the proximal humerus $(n=5)$, proximal femur $(n=3)$, distal femur $(n=16)$, proximal tibia $(n=5)$, distal tibia $(n=1)$, and calcaneus $(\mathrm{n}=2)$. There were three (9\%) Enneking stage I, $16(50 \%)$ stage II, and $13(41 \%)$ stage III patients.

All patients were examined through direct X-ray, computed tomography (CT), and magnetic resonance imaging (MRI) for preoperative surgical planning. All operations were performed by the same experienced tumor surgeon and the operation procedure was similar. A tourniquet was used in all patients if tumor localization allowed. Generally, an adequate longitudinal incision was performed over the lesion to dominate the whole lesion. An oval cortical window was created with a drill and osteotome. The cortical window and affected soft tissue on the cortex were removed. After an extensive curettage was performed, mechanical cleaning was carried out with a highspeed burr. If necessary, the cavity was rinsed with phenol and ethanol solution while preserving the surrounding soft tissue. Then, antibiotic-free bone cement was prepared and the cavity was filled with high viscosity bone cement (Biomet Bone Cement $\mathrm{R}$, Biomet Orthopedics GmbH, Ried, Switzerland). Grooves were created with a scalpel on the surface of cement to increase the cement-graft retention. Thereafter, when the cement was solidified, putty form of DBM (Grafton, Osteotech Inc., Eatontown, NJ, USA) was applied with at least one standard cortical thickness on the cement (Figure 1). Prophylactic osteosynthesis was performed in patients with possible pathological fracture.

Tumor volume [volume $=(4 / 3) \pi(\mathrm{D} 1 / 2)(\mathrm{D} 2 / 2)(\mathrm{D} 3 / 2)]$ was calculated according to the direct preoperative X-rays and CT sections. ${ }^{[4]}$ Patients were routinely controlled with direct radiography every three months for the first year. To assess tumor recurrence and bone regeneration on the cortical window, all patients were evaluated with CT scans in the first postoperative year (Figure 2). As there is no defined classification method in the literature, we used our own methodology to classify the amount of new bone formation on the cortical window regarding $\mathrm{CT}$ scans (Table I). Every measurement on radiological
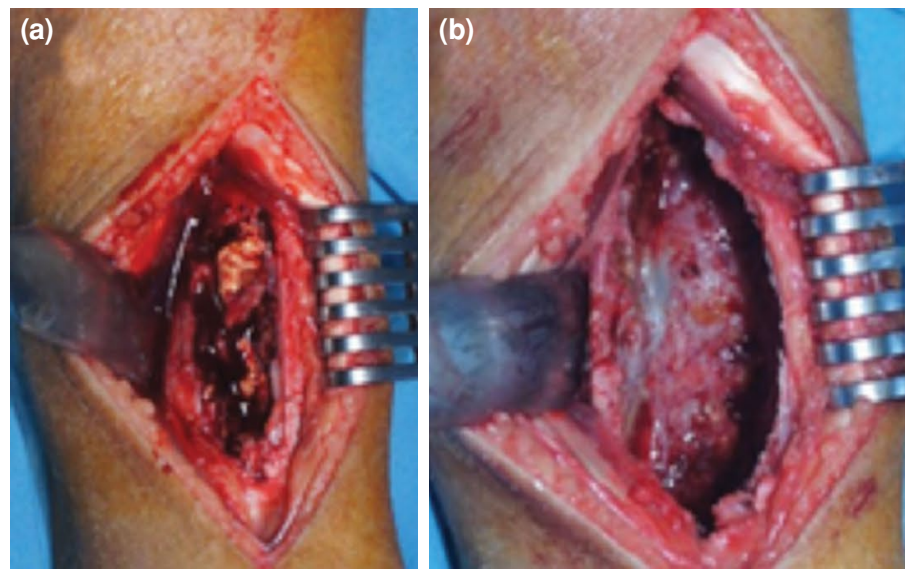
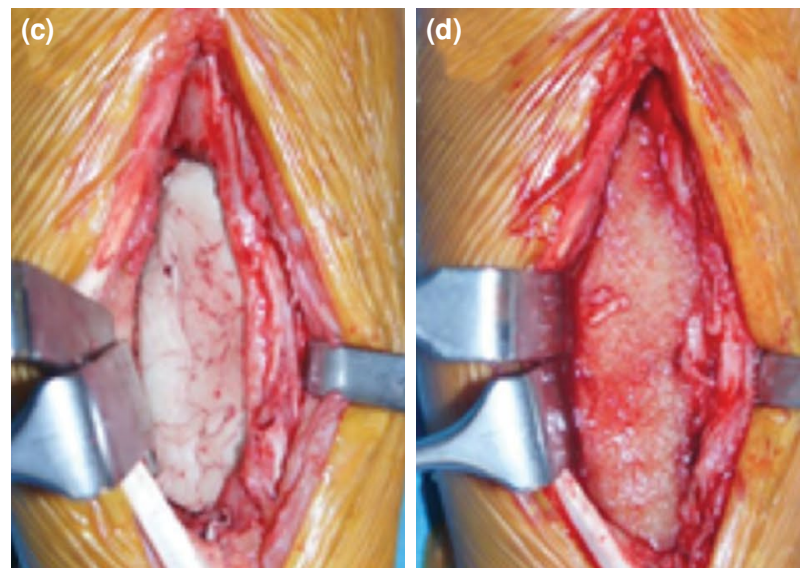

FIGURE 1. (a) Appearance of tumor after cortical window removal. (b) Bone defect following curettage and burr application. (c) Filled bone defect with cement. (d) View after application of demineralized bone matrix minimally one bone cortex thickness on cement. 

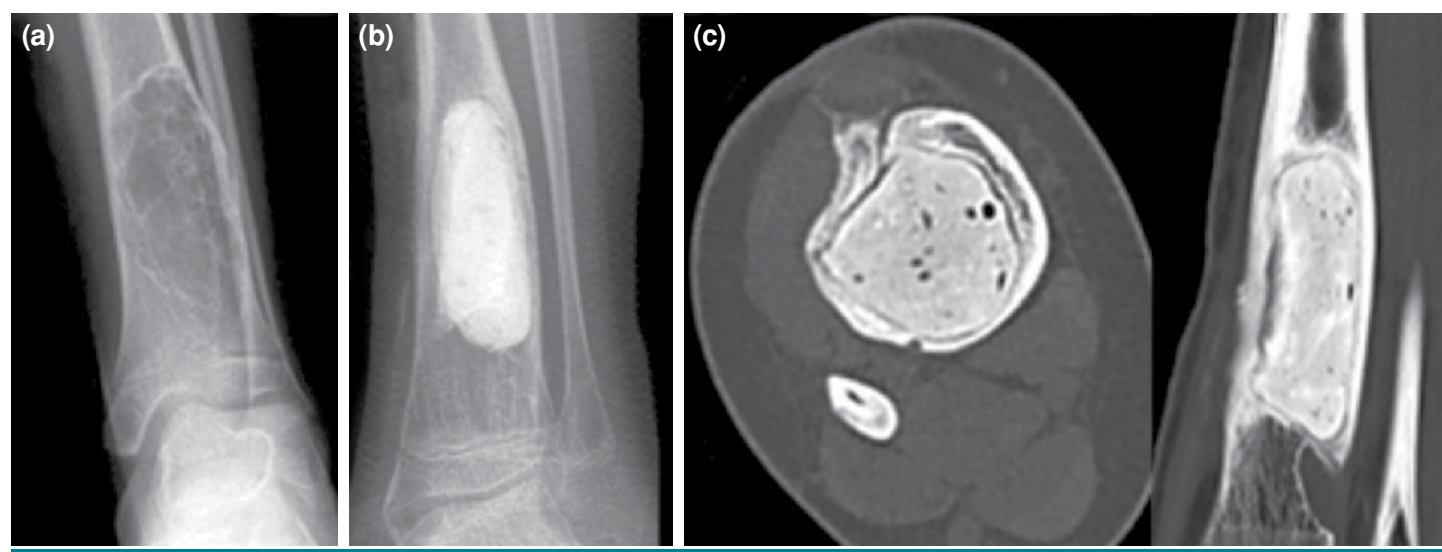

FIGURE 2. (a) Preoperative X-ray image. (b) Postoperative X-ray image. (c) Postoperative axial and coronal computed tomography sections.

images was performed by a radiologist three times to reduce the dating error. Musculoskeletal Tumor Society (MSTS) functional scores of all patients were performed in the first postoperative year. ${ }^{[5]}$

The relationship between new bone formation on the cortical window, age, Enneking tumor stage, functional score, time to return work, size of the cortical window $\left(\mathrm{cm}^{2}\right)$, tumor size $\left(\mathrm{cm}^{3}\right)$, and usage of prophylactic fixation were evaluated.

\section{Statistical analysis}

Statistical analysis of the data was performed using the IBM SPSS version 21.0 software (IBM Corp., Armonk, NY, USA). Concordance of the continuous data to normal distribution was tested by ShapiroWilk test. Continuous variables were expressed with median (minimum-maximum) and mean \pm standard deviation values and categorical variables were expressed with frequency (percentage) values. Two group comparisons were performed using the Mann-Whitney U test; independent sample t-test and three group comparisons were performed using the Kruskal-Wallis and one-way analysis of variance tests. The relationship between non-normally

\begin{tabular}{|cc|} 
TABLE I \\
$\begin{array}{c}\text { Grading system of new bone formation on cortical window } \\
\text { according to postoperative first year computed } \\
\text { tomography scan }\end{array}$ \\
\hline $\begin{array}{cc}\text { New bone formation (\%) } \\
0\end{array}$ & Grading \\
\hline $1-25$ & 0 \\
$26-50$ & 1 \\
$51-75$ & 2 \\
$76-100$ & 3 \\
\end{tabular}

distributed variables was investigated by Spearman's correlation coefficient. Results were reported with 95\% confidence intervals (CI) and related $p$ values. $\mathrm{P}<0.05$ was considered as statistically significant.

\section{RESULTS}

The median size of the cortical window to reach the tumor was $8.3 \mathrm{~cm}^{2}$ (range, 1.6 to $28.4 \mathrm{~cm}^{2}$ ), while the median tumor volume was $17.2 \mathrm{~cm}^{3}$ (range, 2.8 to $139.6 \mathrm{~cm}^{3}$ ). The median time to return to work was 60 days (range, 15 to 220 days). The median new bone formation on the cortical window was grade II. Ten patients' cortical windows were totally healed with the new bone formation (grade IV) and three patients' cortical windows were healed more than a half (grade III) (Figure 3). The median MSTS score was 84.5 (range, 66 to 97). Nine patients (28\%) underwent prophylactic stabilization.

There was no statistically significant difference between tumor size and prophylactic fixation

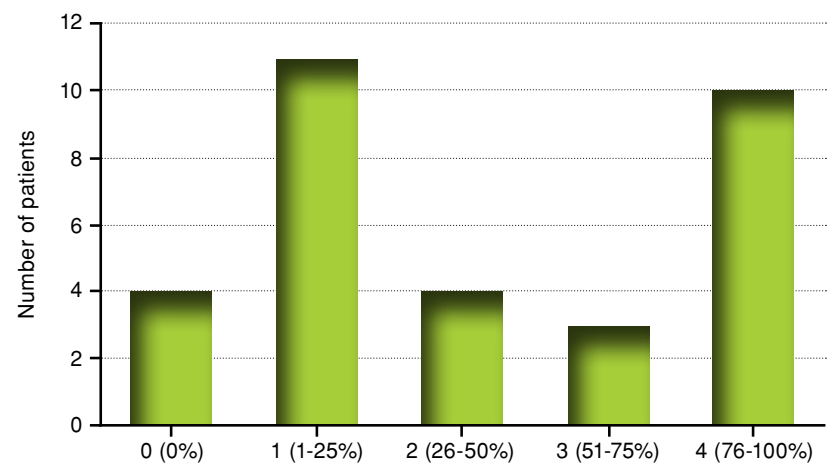

FIGURE 3. Categorization of patients according to grading system. 
$(p=0.592)$. However, there was a statistically significant difference between prophylactic fixation and cortical window $(p=0.013)$. There was no significant difference between the usage of prophylactic fixation and new bone formation on the cortical window $(p=0.967)$. Postoperative first year MSTS score was found statistically worse in patients with prophylactic fixation $(\mathrm{p}<0.001)$.

There was a weak negative correlation between age and new bone formation $(\mathrm{p}=0.046, \mathrm{r}=-0.356)$. There was a moderate negative correlation between the return time to work and MSTS score $(p=0.004$, $\mathrm{r}=-0.498$ ). There was a moderate negative correlation between cortical window and MSTS score $(\mathrm{p}=0.001$, $\mathrm{r}=-0.577)$. There was no correlation between age and MSTS score $(p=0.223)$, tumor volume and MSTS score $(p=0.771)$, new bone formation and cortical window size $(\mathrm{p}=0.692)$, new bone formation and MSTS score $(\mathrm{p}=0.964)$, the return time to work and new bone formation on cortical window $(\mathrm{p}=0.398)$.

Local recurrence happened only in one patient, who had giant cell bone tumor. After aggressive curettage, revision surgery was performed by applying DBM over the cement, as the same procedure. None of the patients had any other complications such as infection, pathological fracture, seroma or hematoma.

\section{DISCUSSION}

The usage of cement in bone tumors provides immediate structural support to bone and absorbing stress. Thermal cytotoxic effect of the cement reduces the local recurrence. ${ }^{[6]}$ Cement reconstruction is a successful method in bone tumors because it provides mechanical support and reduces the possibility of pathological fracture. Therefore, it is more preferable than graft in the load bearing, high stress regions, and large defects. ${ }^{[7]}$ Its simple reconstruction procedure, satisfactory functional and radiological results as well as low-cost increase the range of usage. ${ }^{[8]}$

Demineralized bonematrixis analternativeallograft product for filling bone defects, which provides bone regeneration mainly through osteoconduction and partly osteoinduction. ${ }^{[9]}$ In recent years, the treatment of benign bone tumors with DBM has become popular in orthopedic and maxillofacial surgery due to its high recovery and low complication rates. ${ }^{[10,11]}$ Therefore, it has increased its combined use of other graft materials. There are two level $3^{[12,13]}$ and three level 4 studies, ${ }^{[14-16]}$ which show that combined DBM and autologous bone marrow use is effective in the treatment of active bone cysts. Successful results have been also shown with the use of DBM in combination with steroids. ${ }^{[17]}$ Teng et al. ${ }^{[18]}$ reported that the combined use of allograft and cement in giant-cell bone tumors around the knee has led to less mechanical failure and they suggest this method as an optimal reconstruction strategy. In the literature review, we could not find any data about the combined use of DBM and cement as well as the effect of new bone formation in cortical window on functional scores. ${ }^{[19]}$ By the cement combined DBM treatment, we provide initial mechanical strength by taking advantage of the load-bearing effect of the cement which makes early weight bearing possible. Moreover, we optimize the cost effectivity and reduce the possibility of graft resorption and fracture by using less DBM. In addition, we also increase cortical bone formation that carries the load on removed cortical window. In our study, we found that $3 / 32$ patients had more than $50 \%$ new bone formation on the cortical window and the cortical window of 10/32 patients were almost completely healed with new bone in the first year CT scans. Although the efficacy of DBM to produce live bone is best demonstrated only by histological examination, the thin rim layer, which appears in tomography and direct graphs, shows an increased activity in the scintigraphy. The radiological and histological results are parallel to each other in experimental studies. ${ }^{[20]}$ We did not find any correlation between the functional scores and new bone formation.

It is known that the effectivity of different brands of DBMs varies from each other. ${ }^{[21]}$ The reasons of different results have been shown such as the sterilization process, washing procedure, varying from donor to donor resulting in differences between products, inherent BMP types, and different amounts of graft. ${ }^{[22,23]}$ The fact that the US Food and Drug Administration (FDA) is not performing standard controls for DBMs was shown to be not a surprise in the diversity of DBM results. We think that we have standardized and optimized our treatment, because we used same brand, which has shown superiority, and same form DBM. ${ }^{[21]}$

Traditionally, aging decreases mesenchymal cell differentiation, collagen activity, bone metabolism, and recipient aging declines the effect of allografts. ${ }^{[23]}$ Also, there is an increased risk of non-union with elder population in DBM-treated patients with lumbar fusion. ${ }^{[24]}$ We found a negative correlation between aging and new bone formation on cortical window consistent with the literature.

Prophylactic osteosynthesis is indicated to reduce the possibility of pathological fracture for bone tumors greater than $60 \mathrm{~cm}^{3}$ and in the load bearing areas. ${ }^{[25,26]}$ We did not find any correlation between 
tumor volume and prophylactic fixation. However, no pathological fracture was seen in any patient. In addition, we found a correlation between cortical window size and the use of prophylactic fixation. The literature is unclear and open to research about the relationship between the size of cortical window and the need for prophylactic fixation. We believe that the complication of pathological fracture can be prevented by this surgical technique using the mechanical effect of cement and the biological effect of DBM. We found that functional results were worse in patients undergoing prophylactic fixation. However, it should not be disregarded that the functional outcomes are relatively worse in a possible pathological fracture. ${ }^{[25]}$

This study has some limitations. First, removal of different types of tumors would affect recurrence, time to return to work, and new bone formation on the cortical window. Second, the distribution of the load on the lower and upper extremities could not be the same; therefore, this may have affected the return to work, new bone formation on the cortical window, and functional scores.

In conclusion, the cement combined DBM treatment is a cost-effective, alternative method in tumor surgery, that provides immediate stability and stimulates new bone formation on cortical window. Although new bone formation is achieved on cortical window with this method, new bone formation has not been found to create a change in functional results. The transformation of the new bone to the cortical bone and how long it lasts are open to research. We believe that the histological evaluation of this method supported by controlled studies will guide future tumor treatment methods.

\section{Declaration of conflicting interests}

The authors declared no conflicts of interest with respect to the authorship and/or publication of this article.

\section{Funding}

The authors received no financial support for the research and/or authorship of this article.

\section{REFERENCES}

1. Nogueira Drumond JM. Benign bone tumors and tumorlike bone lesions: Treatment update and new trends. Rev Bras Ortop 2015;44:386-90.

2. Webb JC, Spencer RF. The role of polymethylmethacrylate bone cement in modern orthopaedic surgery. J Bone Joint Surg [Br] 2007;89:851-7.

3. Hass HJ, Krause H, Kroker S, Wagemann W. Implantation of human demineralized bone matrix (DBM) for the treatment of juvenile bone cysts. Oper Orthop Traumatol 2006;18:19-33.

4. Somville J, De Beuckeleer L, De Schepper A, Verstreken J, Taminiau A. Reliability of measuring volume by different methods for tumors of the musculoskeletal system. Acta Orthop Belg 2001;67:338-43.

5. Enneking WF, Dunham W, Gebhardt MC, Malawar M, Pritchard DJ. A system for the functional evaluation of reconstructive procedures after surgical treatment of tumors of the musculoskeletal system. Clin Orthop Relat Res 1993;286:241-6.

6. Martinez, M, Hwang, J, Beebe KS. Local adjuvants for benign aggressive bone tumors, Current Orthopaedic Practice 2014;25:573-9.

7. Szalay K, Antal I, Kiss J, Szendroi M. Comparison of the degenerative changes in weight-bearing joints following cementing or grafting techniques in giant cell tumour patients: medium-term results. Int Orthop 2006;30:505-9.

8. Özer D, Er T, Aycan OE, Öke R, Coşkun M, Kabukçuoğlu YS. May bone cement be used to treat benign aggressive bone tumors of the feet with confidence? Foot (Edinb) 2014;24:1-5.

9. Urist MR, Strates BS. Bone formation in implants of partially and wholly demineralized bone matrix. Including observations on acetone-fixed intra and extracellular proteins. Clin Orthop Relat Res 1970;71:271-8.

10. Drosos GI, Touzopoulos P, Ververidis A, Tilkeridis K, Kazakos K. Use of demineralized bone matrix in the extremities. World J Orthop 2015;6:269-77.

11. Dinopoulos H, Dimitriou R, Giannoudis PV. Bone graft substitutes: What are the options? Surgeon 2012;10:230-9.

12. Di Bella C, Dozza B, Frisoni T, Cevolani L, Donati D. Injection of demineralized bone matrix with bone marrow concentrate improves healing in unicameral bone cyst. Clin Orthop Relat Res 2010;468:3047-55.

13. Park IH, Micic ID, Jeon IH. A study of 23 unicameral bone cysts of the calcaneus: open chip allogeneic bone graft versus percutaneous injection of bone powder with autogenous bone marrow. Foot Ankle Int 2008;29:164-70.

14. Kanellopoulos AD, Yiannakopoulos CK, Soucacos PN. Percutaneous reaming of simple bone cysts in children followed by injection of demineralized bone matrix and autologous bone marrow. J Pediatr Orthop 2005;25:671-5.

15. Docquier PL, Delloye C. Treatment of aneurysmal bone cysts by introduction of demineralized bone and autogenous bone marrow. J Bone Joint Surg [Am] 2005;87:2253-8.

16. Rougraff BT, Kling TJ. Treatment of active unicameral bone cysts with percutaneous injection of demineralized bone matrix and autogenous bone marrow. J Bone Joint Surg [Am] 2002;84:921-9.

17. Sung AD, Anderson ME, Zurakowski D, Hornicek FJ, Gebhardt MC. Unicameral bone cyst: a retrospective study of three surgical treatments. Clin Orthop Relat Res 2008;466:2519-26.

18. Teng W, Lin P, Li Y, Yan X, Li H, Li B, et al. Bone combined cement grafting in giant cell tumor around the knee reduces mechanical failure. Int Orthop 2019;43:475-82.

19. Atik OŞ. Which articles do we prefer to publish?. Eklem Hastalik Cerrahisi 2018;29:1.

20. Enneking WF, Campanacci DA. Retrieved human allografts: a clinicopathological study. J Bone Joint Surg [Am] 2001;83:971-86.

21. Peterson B, Whang PG, Iglesias R, Wang JC, Lieberman JR. Osteoinductivity of commercially available demineralized bone matrix. Preparations in a spine fusion model. J Bone Joint Surg [Am] 2004;86:2243-50. 
22. Sammarco VJ, Chang L. Modern issues in bone graft substitutes and advances in bone tissue technology. Foot Ankle Clin 2002;7:19-41.

23. Mueller SM, Glowacki J. Age-related decline in the osteogenic potential of human bone marrow cells cultured in threedimensional collagen sponges. J Cell Biochem 2001;82:583-90.

24. Ajiboye RM, Eckardt MA, Hamamoto JT, Sharma A, Khan AZ, Wang JC. Does Age Influence the Efficacy of Demineralized Bone Matrix Enriched with Concentrated Bone Marrow Aspirate in Lumbar Fusions?. Clin Spine Surg
2018;31:E30-E5.

25. Perisano C, Barone C, Stomeo D, Di Giacomo G, Vasso M, Schiavone Panni A, et al. Indications for prophylactic osteosynthesis associated with curettage in benign and low-grade malignant primitive bone tumors of the distal femur in adult patients: a case series. J Orthop Traumatol 2016;17:377-82.

26. Kornah B, Safwat H, Ghany TA, Aal MA, Saleem N. Prophylactic Fixation of Impending Fractures. MOJ Orthop Rheumatol 2016;6:00206. 